\title{
DEMANDAS ESTRUCTURALES INDUCIDAS POR VIENTOS HURACANADOS Y TERREMOTOS EN UN EDIFICIO FLEXIBLE EN la República Dominicana
}

\author{
Structural demands induced by hurricane winds and \\ earthquakes on a flexible building in the Dominican Republic
}

\author{
Kalil Erazo ${ }^{a}$ y Adela Taveras ${ }^{b}$ \\ Recibido: $14 / 6 / 2021 \bullet$ Aprobado: 21/6/2021
}

\begin{abstract}
Cómo citar: Erazo, K., \& Taveras, A. (2021). Demandas estructurales inducidas por vientos huracanados y terremotos en un edificio flexible en la República Dominicana. Ciencia, Ingenierías y Aplicaciones, 4(1), 57-78. DOI: https://doi.org/10.22206/cyap.2021.v4i1.pp57-78
\end{abstract}

\section{Resumen}

Este articulo presenta los resultados de un análisis comparativo de las demandas estructurales inducidas por vientos huracanados y terremotos en un edificio flexible diseñado para la ciudad de Santo Domingo en la República Dominicana. La estructura consiste en un edificio de 33 niveles con periodos fundamentales de vibración mayores de 3 segundos en las dos principales direcciones ortogonales, por lo que se considera un edificio flexible. El edificio fue inicialmente diseñado para cumplir con los requerimientos de la normativa vigente para efectos inducidos por terremotos (R-001) sin tomar en consideración los efectos inducidos por vientos huracanados; subsecuentemente, las demandas inducidas por viento fueron estimadas usando el procedimiento direccional del ASCE 07-10, utilizando como base la velocidad de diseño en recientes mapas de amenaza de viento desarrollados para la región del Caribe. Los resultados muestran que para la estructura analizada los efectos de vientos son significativamente mayores a los efectos sísmicos, lo que evidencia la urgencia de actualizar la actual normativa que define las cargas minimas de diseño por viento de la República Dominicana. La justificación de dicha urgencia es el auge en la construcción de estructuras flexibles (principalmente edificios esbeltos) cuyo comportamiento tiende a ser

\footnotetext{
a Profesor Investigador. Área de Ingeniería. Instituto Tecnológico de Santo Domingo (INTEC). Correo-e: Kalil.Erazo@intec.edu.do

b Asistente de Investigación. Área de Ingeniería. Instituto Tecnológico de Santo Domingo (INTEC). Correo-e: adela_tavera_urena@hotmail.com
} 
dominado por efectos inducidos por viento, en especial en regiones de alta recurrencia de huracanes. Las demandas estructurales comparadas incluyen desplazamientos laterales, derivas de entrepiso, cortante basal y momento de vuelco.

Palabras clave: vientos huracanados; procedimiento direccional ASCE 07; cargas sísmicas; cargas de viento; edificios flexibles.

\begin{abstract}
This article presents a comparative analysis of the structural demands induced by hurricane winds and earthquakes on a flexible building designed for Santo Domingo in the Dominican Republic. The structure consists in a 33-story building with fundamental vibration periods larger than 3 seconds in the two main orthogonal directions, and thus considered flexible. The building was initially designed to comply with the requirements of the local seismic building code (R-001) without considering the effects induced by hurricane winds; subsequently the demands induced by wind were estimated using the ASCE 07-10 Directional Procedure with the base design wind speed obtained from recent wind hazard maps developed for the Caribbean region. The results show that for the analyzed structure the wind effects are significantly larger than the seismic effects, which demonstrates the urgency to update the minimum design wind loads building code of the Dominican republic. The justification for this urgency is the increase in the number of flexible structures (mainly slender buildings) whose behavior is dominated by the effects induced by wind, specially in regions with a high hurricane recurrence. The structural demands compared include lateral displacements, inter-story drifts, base shear and overturning moment.
\end{abstract}

Keywords: hurricane winds; ASCE 07 directional procedure; seismic loads; wind loads; flexible buildings. 


\section{Introducción}

Las infraestructuras civiles forman una de las bases indispensables para el desarrollo económico sostenible, el bienestar y la calidad de vida de toda sociedad (Rozas \& Sánchez, 2004). Infraestructuras como puentes, presas, hospitales, viviendas, sistemas de energía y comunicación son necesarias para satisfacer las necesidades que demanda la población para subsistir y desarrollarse. Estas infraestructuras deben ser diseñadas para resistir de manera confiable las solicitaciones de carga esperadas durante su vida útil.

Dentro de los diferentes eventos que inducen efectos inerciales (dinámicos) considerables en infraestructuras civiles se destacan terremotos y huracanes (López \& Martínez, 2003; Neris, Domínguez, Pérez, Rodríguez \& Cano, 2010). Los efectos inducidos por dichos eventos dependen tanto de las características de las fuerzas (principalmente magnitud, dirección y contenido de frecuencia) como de las características físicas de la estructura y del medio que la rodea. Las características físicas de una estructura y el medio que la rodea definen las propiedades dinámicas de la misma y de las cuales depende el comportamiento durante eventos en los cuales efectos inerciales son importantes. Las principales propiedades dinámicas que definen dicho comportamiento son amortiguamiento, frecuencias y modos de vibración.

Dependiendo de las principales frecuencias de vibración de una estructura (con mayor factor de participación modal), las estructuras se consideran flexibles si su frecuencia fundamental de vibración es menor de $1 \mathrm{~Hz}$, y rígidas en caso contrario. En el caso de las estructuras rígidas, sus propiedades dinámicas son tal que generalmente los efectos inducidos por terremotos son más importantes que los efectos inducidos por vientos huracanados debido a la amplificación dinámica, es decir, el hecho de que el contenido de frecuencia con mayor energía en el movimiento de la base tiende a concentrarse en frecuencias mayores de $1 \mathrm{~Hz}$. Similarmente, en el caso de estructuras flexibles, los efectos inducidos por vientos huracanados tienden a dominar el diseńo, debido a que el contenido de frecuencia con mayor energía se concentra en frecuencias menores de $1 \mathrm{~Hz}$. Esto se ilustra esquemáticamente en la figura 1. 


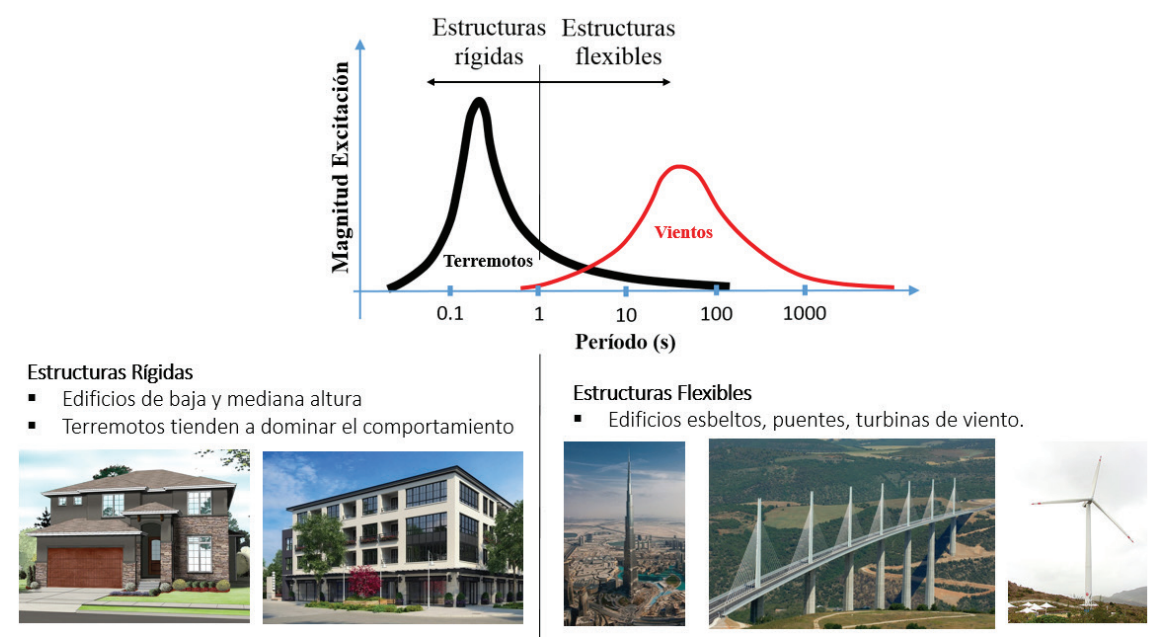

Figura 1. Rangos de importancia de los efectos de terremotos y vientos huracanados en estructuras rígidas y flexibles

El objetivo de este artículo es estimar las demandas inducidas tanto por terremotos como huracanes en una estructura flexible diseñada para la ciudad de Santo Domingo (República Dominicana). Debido a su ubicación geográfica, la República Dominicana presenta un alto peligro tanto para terremotos como huracanes (Ministerio de Economía, Planificación y Desarrollo, 2012). El interés del artículo radica en dos razones principales: i) el incremento importante en la construcción de estructuras flexibles, principalmente edificios esbeltos de más de 30 niveles; $y$ ii) la carencia de una normativa actualizada para considerar los efectos de viento en el diseño de estructuras civiles.

La importancia de estudiar los efectos dinámicos inducidos por el viento en estructuras flexibles se volvió inminente a raíz del colapso del puente de Tacoma Narrows en el estado de Washington (Estados Unidos) en el año 1940. El colapso del puente, luego de ser sometido a efectos dinámicos inducidos por el viento, es mostrado en la figura 2. El colapso se debió a una inestabilidad aeroelástica conocida como flameo (flutter en inglés). Esta inestabilidad se caracteriza por deformaciones excesivas de torsión y flexión con frecuencias de vibración similares, lo cual resultó en la transferencia de energía del flujo de viento a la estructura (Billah \& Scanlan, 1990). Durante el flameo la 
estructura tiene el potencial de perder su capacidad de disipar energía, y su amortiguamiento se puede volver nulo o incluso negativo.

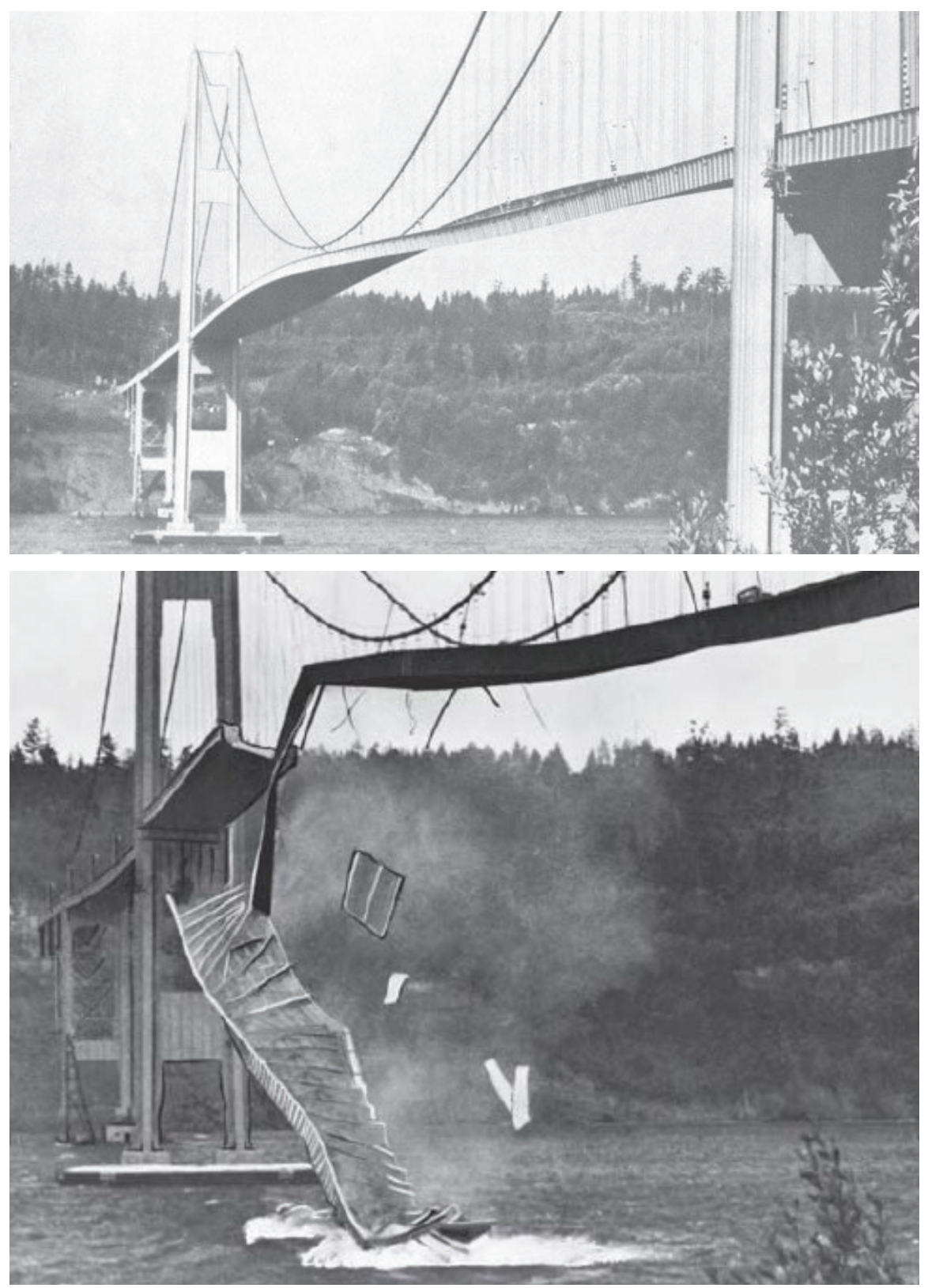

Figura 2. Colapso del puente Tacoma Narrows debido a efectos aerodinámicos inducidos por viento 
El colapso del puente Tacoma Narrows reveló la importancia del estudio de los efectos dinámicos e inestabilidades aeroelásticas en infraestructuras flexibles sometidas a los efectos inducidos por el viento, y sirvió para impulsar la investigación en aerodinámica e ingeniería de viento de manera importante. El resultado de estas investigaciones ha influenciado el diseño de puentes y otras estructuras flexibles desde el año 1940 hasta la fecha, incluyendo la incorporación a las normativas internacionales el requerimiento de análisis avanzados cuando la estructura lo amerita.

\section{Amenazas de vientos huracanados y terremotos en la República Dominicana}

Debido a su ubicación geográfica, las dos principales amenazas naturales que dominan el diseño de infraestructuras civiles en la República Dominicana son terremotos y huracanes. En el caso de terremotos, la amenaza se debe a distintas fallas sísmicas activas mostradas en la figura 3.

De dichas fallas cabe destacar la falla Septentrional en la región norte. Basado en mediciones de la deformación de la falla obtenidas mediante un sistema de posicionamiento global, la falla Septentrional presenta niveles de deformación y energía acumulada con capacidad de generar un terremoto característico de magnitud 7.8 (Calais et al., 2002). La falla Septentrional es una falla de desplazamiento horizontal (transformante o de desgarre) que cruza la región norte de La Española y acomoda parte del movimiento entre las placas de Norte América y el Caribe; cabe recalcar que este movimiento entre dichas placas ocasionó el devastador terremoto de Haití de 2010. La falla Septentrional constituye la mayor fuente de peligro sísmico para infraestructuras civiles en la región Norte (Erazo, 2019; 2020).

Otras fallas sísmicas activas importantes incluyen la falla de Neiba y la falla Enriquillo, esta última causante del devastador terremoto de Haití de 2010, el cual dejó un saldo de más de 300,000 fallecidos siendo considerado como una de las catástrofes humanas más graves de la historia (Neris et al., 2010). 


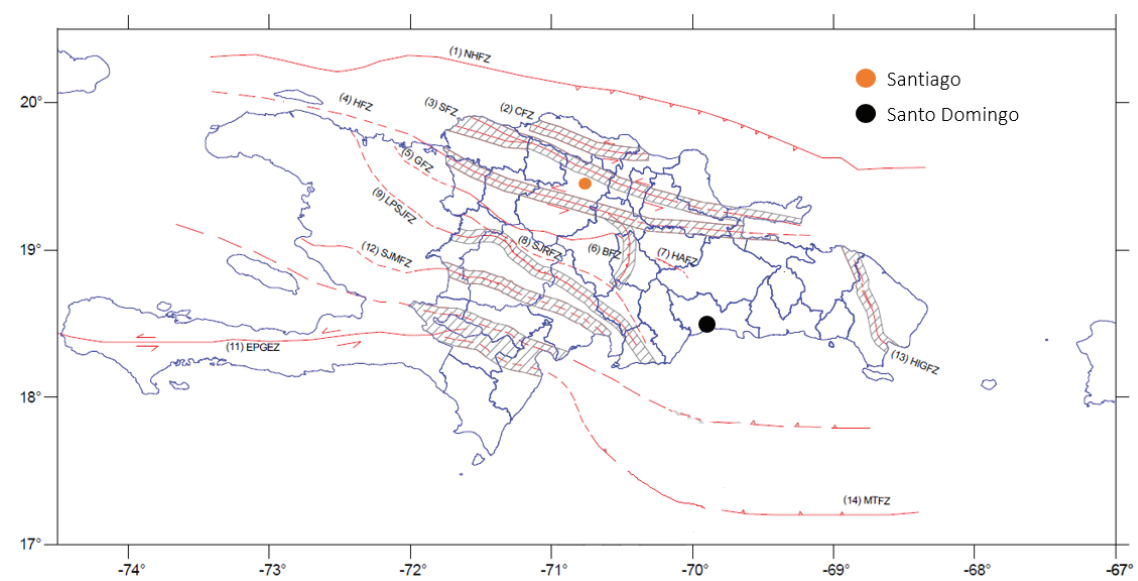

Figura 3. Fallas sísmicas de La Española; la falla Septentrional se denota como "SFZ" (Reglamento para el análisis y diseño sísmico de estructuras R-001, 2011)

La segunda fuente de peligro considerable debido a eventos naturales extremos se debe a la ocurrencia de huracanes. La República Dominicana se encuentra en el centro del cinturón de huracanes, una zona caliente del Océano Atlántico que inicia en la costa oeste de África hasta el este de América Central y el Golfo de México. La figura 4 muestra la trayectoria de huracanes en el Atlántico entre 1851 y 2019.

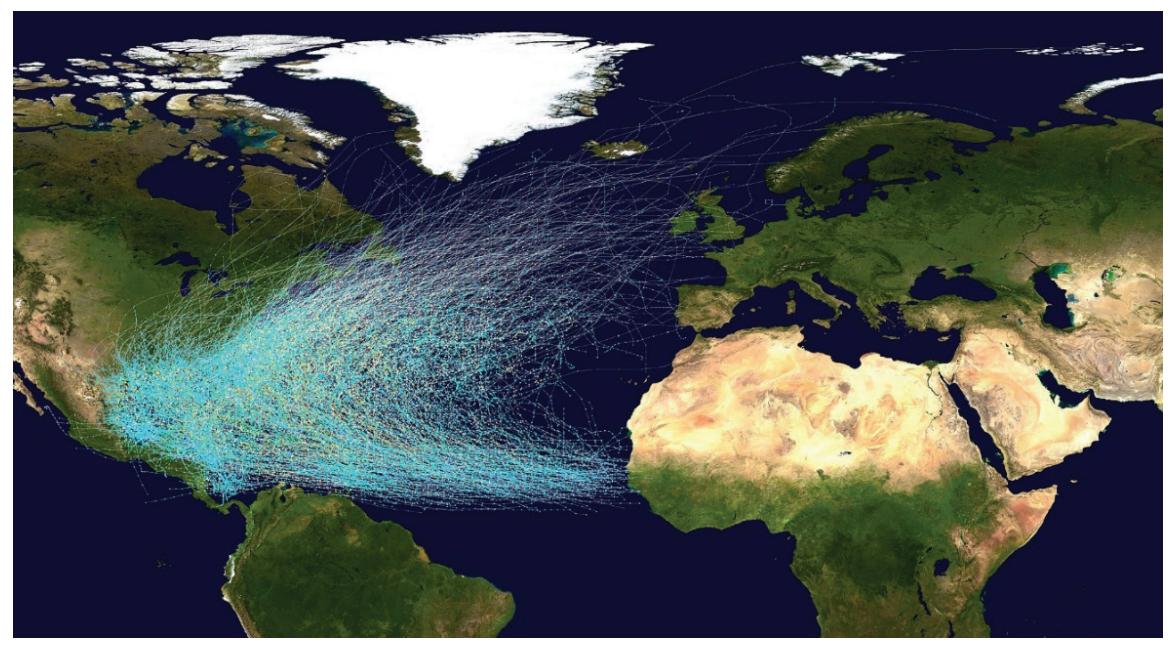

Figura 4. Trayectoria de huracanes en el Atlántico entre 1851 y 2019 
Los huracanes son eventos que tienen la capacidad de generar efectos dinámicos debido a vientos extremos en estructuras, y se pueden clasificar de acuerdo a la escala Saffir-Simpson. De principal importancia son los huracanes de magnitud 3 a 5 , los cuales tienen la capacidad de generar daños y pérdidas importantes. La tabla 1 muestra los principales huracanes que han afectado a la República Dominicana de manera directa en los últimos 85 años. Se puede observar la alta recurrencia de huracanes de gran magnitud, que han provocado pérdidas humanas y económicas significativas.

Tabla 1. Principales eventos climáticos extremos recientes en la República Dominicana

\begin{tabular}{ccc}
\hline Nombre & Fecha & Consecuencias \\
\hline $\begin{array}{c}\text { Tormenta tropical } \\
\text { Olga }\end{array}$ & 2007 & 37 fallecidos debido a la operación de un embalse \\
en Santiago
\end{tabular}


Cabe destacar que el último huracán con vientos extremos en afectar la República Dominicana de manera directa fue el huracán Georges (categoría 4 en la escala de Saffir-Simpson) en el año 1998. Desde este año el medio construido de este país ha cambiado de manera importante, y la proporción de las infraestructuras flexibles con alta vulnerabilidad ante vientos extremos se ha incrementado significativamente. Esto se debe al auge en la construcción de edificaciones susceptibles a ser afectadas por los efectos del viento, tales como edificios esbeltos de gran altura, estructuras flexibles de acero y puentes de grandes luces.

\section{Caso de estudio}

El objetivo de este artículo es comparar las demandas de vientos huracanados y terremotos en un edificio flexible. Con el fin de analizar la importancia de los efectos del viento y para definir un punto de partida de dicha comparación, se diseñó la estructura para satisfacer los requerimientos de carga viva, carga muerta y carga sísmica de acuerdo al código de diseńo vigente R-001. Luego se procedió a analizar la estructura para cargas de viento usando el método direccional del ASCE-07. El objetivo es determinar las demandas inducidas por viento cuando las mismas no son consideradas en el diseño de la estructura.

Cabe destacar que la República Dominicana no cuenta con un código de diseño o construcción actualizado que incorpore una metodología para definir las cargas mínimas de diseño por viento, y el único documento oficial es una guía de los años 80 (Ministerio de Obras Públicas y Comunicaciones, 1980).

\subsection{Descripción de la estructura}

La estructura de estudio consiste en un edificio residencial/comercial de 33 niveles, el cual forma parte de un proyecto inmobiliario a desarrollarse en la ciudad de Santo Domingo, y el modelo se basa en el diseño arquitectónico elaborado por una firma de arquitectos. El modelo de la estructura se muestra en la figura 5. 

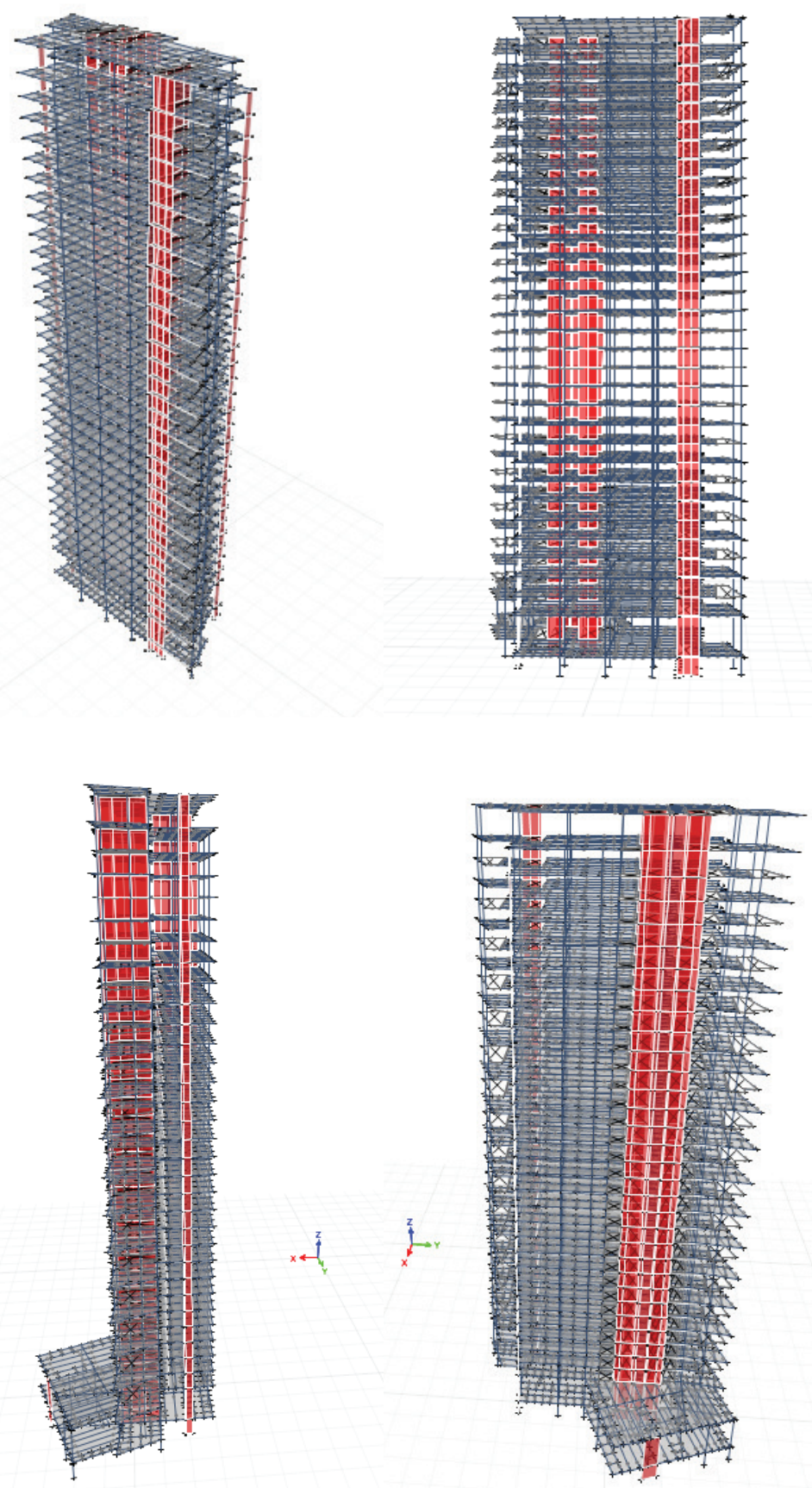

Figura 5. Vistas tridimensionales del modelo computacional del edificio 

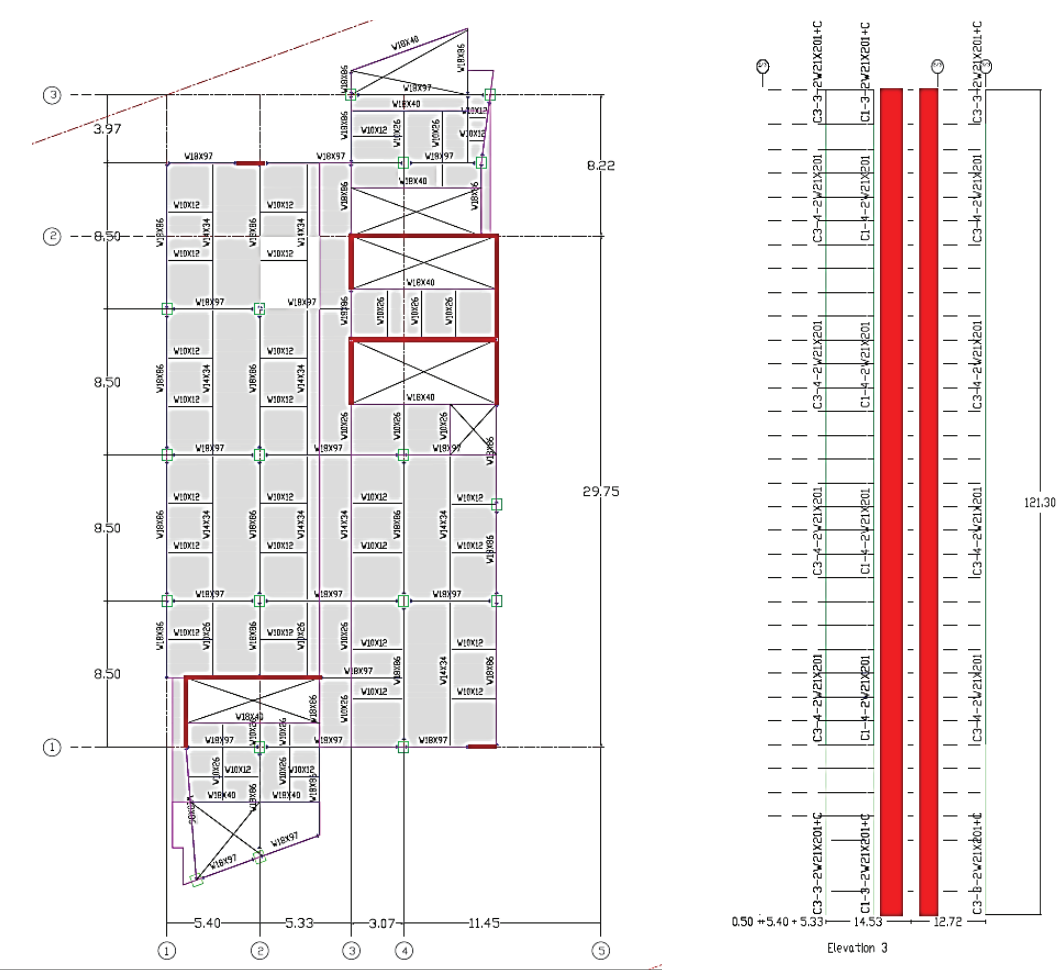

Figura 6. Vista en planta (izquierda) y elevación del edificio (derecha). Dimensiones en metros

La figura 6 (izquierda) muestra la planta típica del edificio y una elevación (derecha). La altura total es de $121.3 \mathrm{~m}$. El sistema estructural consiste en un sistema combinado de pórticos especiales de acero y muros de concreto reforzado. Las columnas de acero consisten en secciones compuestas formadas por vigas de acero A50 soldadas para formar una sección cerrada, similar a una sección tipo HSS. Los muros de concreto reforzado son de sección variable entre $0.30 \mathrm{~m}$ y $0.60 \mathrm{~m}$ de espesor con resistencias entre $300 \mathrm{kgf} / \mathrm{cm}^{2}$ y $500 \mathrm{kgf} / \mathrm{cm}^{2}$.

Definidas las características geométricas de la estructura y sus elementos, incluyendo las propiedades de los materiales, condiciones de borde y de conexión, quedan definidas las propiedades mecánicas de la estructura (masa, rigidez y amortiguamiento) las cuales definen a su vez las características de vibración de la misma. La figura 7 presenta los modos de vibración con mayor participación en la 
respuesta dinámica de la estructura. Como se observa, los primeros 6 modos acumulan alrededor del $70 \%$ de la masa modal; el número de modos usados es tal que la masa modal acumulada es de $99 \%$. Los dos modos fundamentales en las dos direcciones principales de la estructura tienen periodos de vibración de 4.83 s (dirección vertical de acuerdo a la vista en planta en la figura 6) y 3.98 s (dirección horizontal de acuerdo a la vista en planta en la figura 6 ).

\begin{tabular}{|l|l|l|l|}
\multicolumn{1}{c}{ Mode } & \multicolumn{2}{c}{$\begin{array}{c}\text { Period } \\
\text { sec }\end{array}$} & \multicolumn{2}{c}{ UX } \\
\hline 1 & 4.827 & 0.0947 & 0.4296 \\
\hline 2 & 3.981 & 0.5264 & 0.1077 \\
\hline 3 & 2.743 & 0.0135 & 0.1296 \\
\hline 4 & 1.236 & 0.0132 & 0.0973 \\
\hline 5 & 0.787 & 0.1458 & 0.0322 \\
\hline 6 & 0.598 & 0.0231 & 0.0396 \\
\hline
\end{tabular}

Figura 7. Periodos y factores de participación modal de los primeros 6 modos de vibración

\subsection{Descripción de las cargas}

Como se mencionó anteriormente, la estructura fue inicialmente diseñada para carga viva, carga muerta y carga sísmica y, posteriormente, se analizaron las demandas inducidas por viento. El objetivo es determinar las demandas por viento y su importancia cuando las mismas no son consideradas en el diseńo de la estructura.

La carga muerta consistió en el peso de elementos estructurales y no-estructurales considerados fijos. El peso total de dichos elementos que componen la estructura es $1.98 \times 10^{4}$ Ton. La carga viva varía por nivel en el rango $200-1000 \mathrm{kgf} / \mathrm{m}^{2}$ de acuerdo al uso (residencial, comercial, parqueos, zonas con equipos pesados considerados movibles). 
Las demandas por carga sísmica se obtuvieron usando un análisis modal espectral, considerando el espectro de diseño para la ciudad de Santo Domingo de acuerdo a las condiciones de sitio de la ubicación del proyecto (Ministerio de Obras Públicas y Comunicaciones, 2011). Se utilizó un factor de reducción por ductilidad de 5. El espectro de diseño (antes de aplicar el factor de reducción por ductilidad) se muestra en el panel izquierdo de la figura 8. El gráfico muestra los dos principales modos de vibración y se observa que los mismos se asocian a valores de pseudo-aceleración espectral relativamente bajos en comparación con el máximo del espectro. El número de modos es tal que la masa modal acumulada es de $99 \%$; las demandas estimadas por los modos fueron combinadas usando el procedimiento CQC.

Las demandas por carga de viento se obtuvieron utilizando el método direccional (Directional Procedure en inglés) del código ASCE 7-10 (American Society of Civil Engineers, 2010). La velocidad básica de diseño (velocidad de ráfaga de 3 segundos a $10 \mathrm{~m}$ de altura) se definió con base en el mapa de amenaza de viento propuesto por Vickery y Wadhera para un periodo de retorno de 1700 años, mostrado en la figura 8 (Vickery \& Wadhera, 2010). Una descripción detallada del procedimiento direccional para cargas de viento aplicado a la estructura de este manuscrito se presentará en otro artículo por los autores.
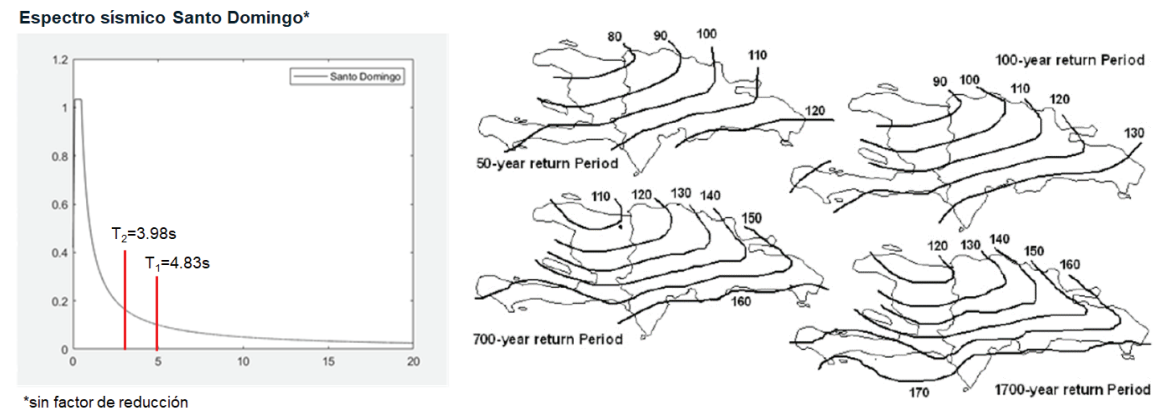

Figura 8. Espectro de diseño en g y velocidad de viento de diseño (ráfaga pico de 3 segundos a una altura de 10 en campo abierto, en millas por hora) 


\section{Resultados}

En esta sección se presentan los resultados de la estimación de las demandas obtenidas utilizando un análisis modal espectral para cargas sísmica y el procedimiento direccional para cargas de viento. Como se mencionó anteriormente, el diseño de la estructura, es decir, la selección de la configuración estructural y las secciones de los distintos elementos se basó en los resultados del análisis sísmico, sin considerar la carga de viento. Con la estructura diseñada para el caso de carga sísmica se procedió a realizar el análisis de cargas viento para determinar las demandas generadas. El objetivo es determinar la importancia de los efectos de la carga de viento si la misma es ignorada durante el análisis y diseño de la estructura.

La figura 9 muestra el cortante basal, el momento de vuelco y la deriva de techo calculadas para sismo y viento en la dirección $\mathrm{x}$ que coincide con la dimensión corta en planta (ver Figura 5). Como se observa, las demandas inducidas por viento exceden de manera significativa las demandas inducidas por terremotos. El cortante basal por viento excede aproximadamente por un factor de 8 el cortante basal debido a terremoto. De manera similar, el momento de vuelco por viento excede el mismo por terremoto por un factor de aproximadamente 11, mientras que la deriva de techo por viento es casi 7 veces la deriva de techo por sismo. La deriva límite de acuerdo al código R-001 es 0.016 para estructuras en las cuales deformaciones laterales no causan daños importantes en componentes no-estructurales. 

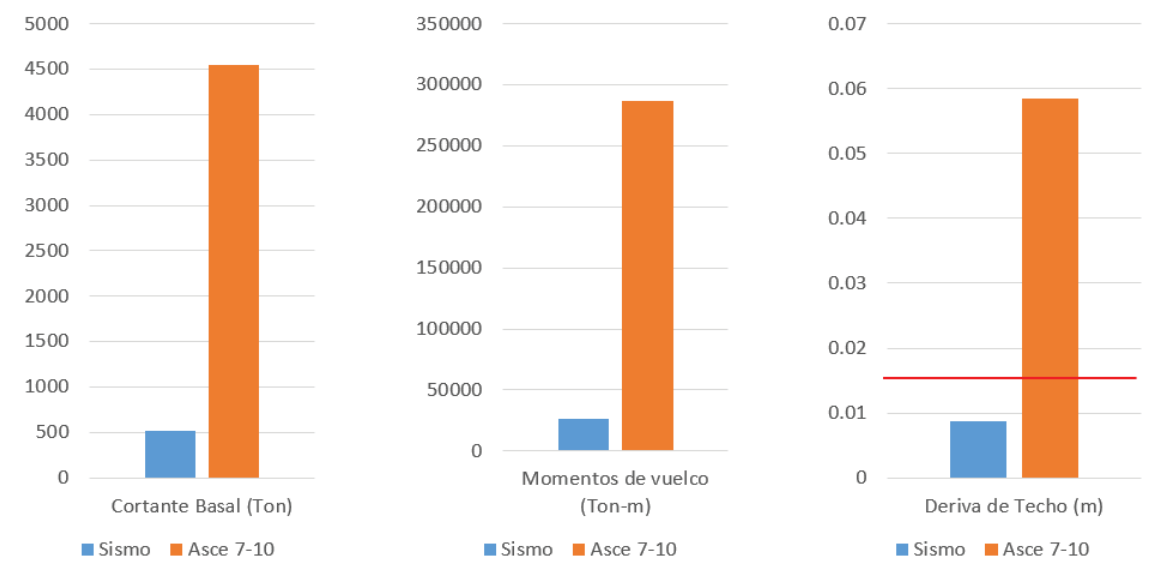

Figura 9. Demandas estructurales inducidas por sismo y viento para análisis en la dirección $x$ (dimensión corta en planta) de acuerdo a la figura 6

Similarmente, la figura 10 muestra las demandas estructurales estimadas en la dirección vertical. Como se observa, las demandas de viento exceden las demandas de sismo por factores entre 3 y 5 .
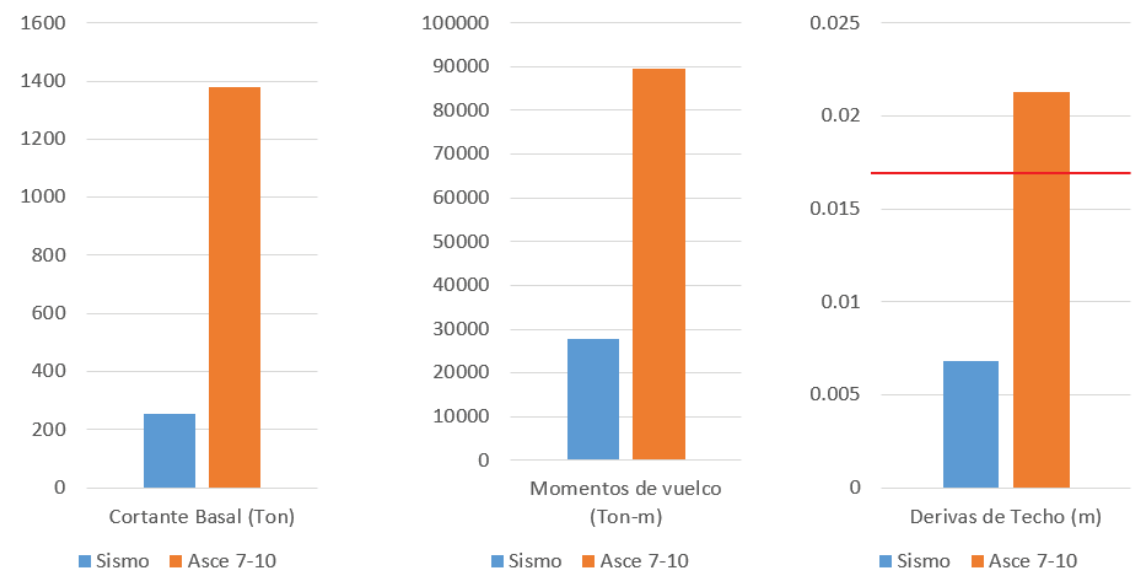

Figura 10. Demandas estructurales inducidas por sismo y viento para análisis en la dirección y (dimensión larga en planta) de acuerdo a la figura 6 
La figura 11 muestra las derivas de entrepiso por nivel para viento (ASCE 7-10) y sismo tanto en la dirección $\mathrm{x}$ (horizontal) como en la dirección y (vertical). Se puede observar que las demandas por viento exceden significativamente las de sismo en todos los niveles, y en la dirección x exceden el límite de 0.016 de la normativa vigente.

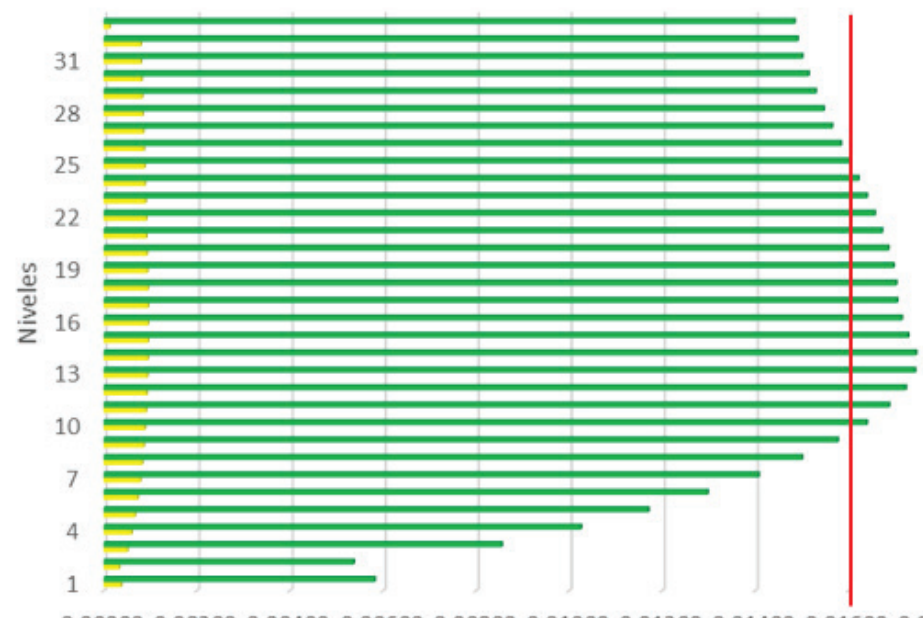

0.000000 .002000 .004000 .006000 .008000 .010000 .012000 .014000 .016000 .01800 Desplazamiento Relativo

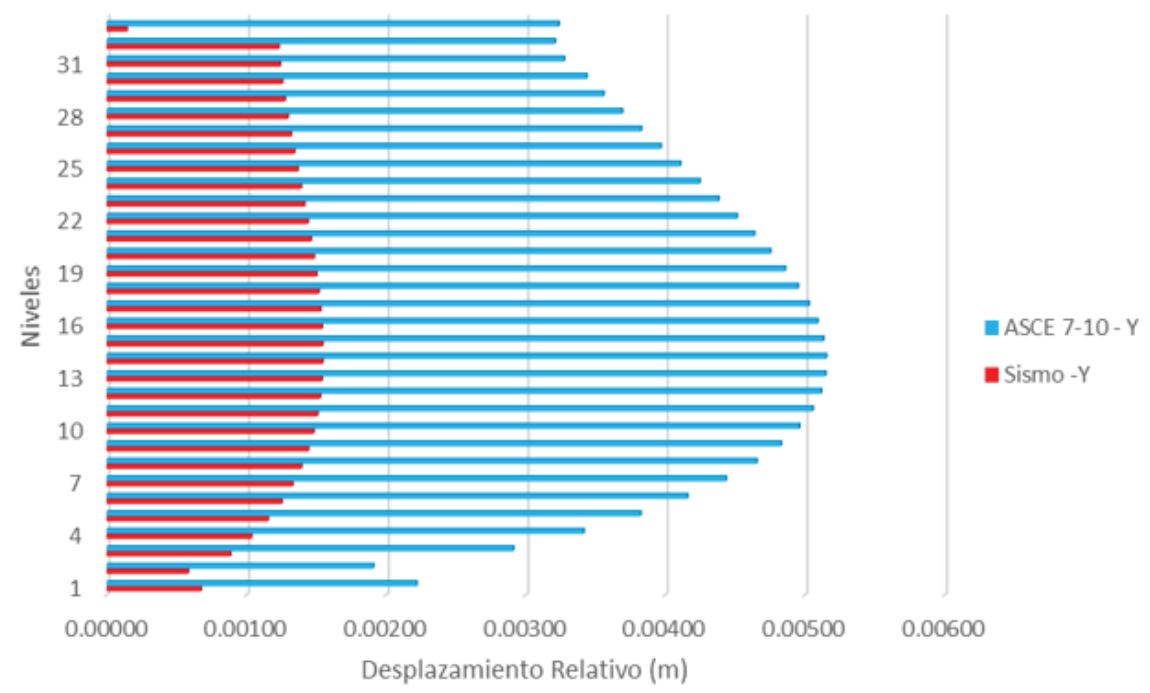

Figura 11. Derivas de entrepiso inducidas por sismo y viento para análisis en la dirección $x$ (horizontal) y la dirección $y$ (vertical) 
Además de la comparación de las cargas inducidas por terremotos y viento estimadas de acuerdo al procedimiento direccional del ASCE 07-10, nos interesa comparar las demandas de viento inducidas por el procedimiento direccional y la normativa vigente para determinar cargas de viento en la República Dominicana conocida como el boletín 9/80. Las figuras 12 y 13 muestran, respectivamente, las demandas de viento estimadas en la dirección $x$ o dimensión corta en planta y dirección $y$ que coincide con la dimensión larga en planta .
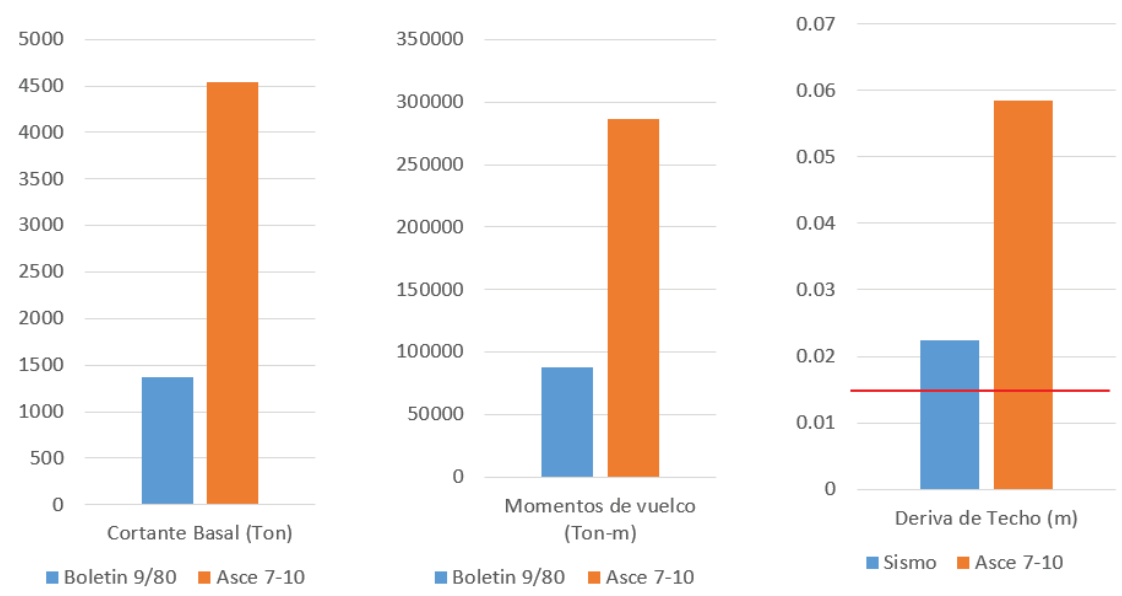

Figura 12. Demandas estructurales en la dirección $x$ (horizontal) inducidas por viento estimadas de acuerdo al procedimiento direccional del ASCE 7-10 y la normativa vigente Boletín 9/80

Como se observa, las demandas estimadas con el procedimiento direccional exceden las demandas estimadas con la normativa vigente por factores de 2-3, lo que muestra la necesidad de actualizar la normativa vigente con carácter de urgencia. 

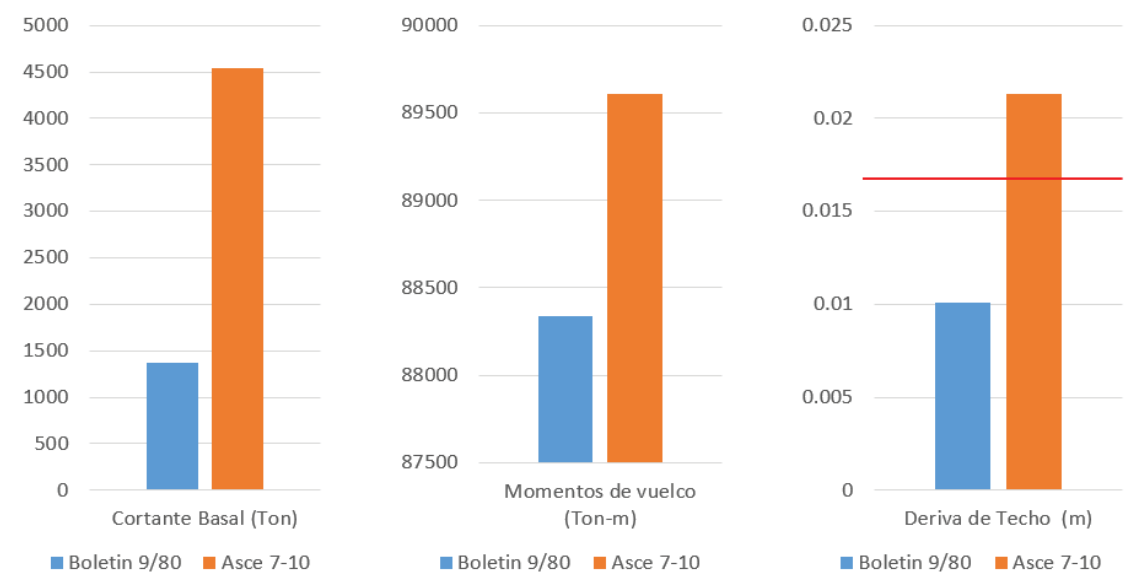

Figura 13. Demandas estructurales en la dirección $y$ (vertical) inducidas por viento estimadas de acuerdo al procedimiento direccional del ASCE 7-10 y la normativa vigente Boletín 9/80

Finalmente, la figura 14 muestra la estimación de las derivas de piso inducidas por viento estimadas tanto de acuerdo al procedimiento direccional como la normativa vigente (Boletín 9/80). Como se observa, las derivas estimadas de acuerdo al procedimiento direccional exceden significativamente las estimadas en la dirección corta (la cual controla el diseńo) de acuerdo a la normativa vigente. 

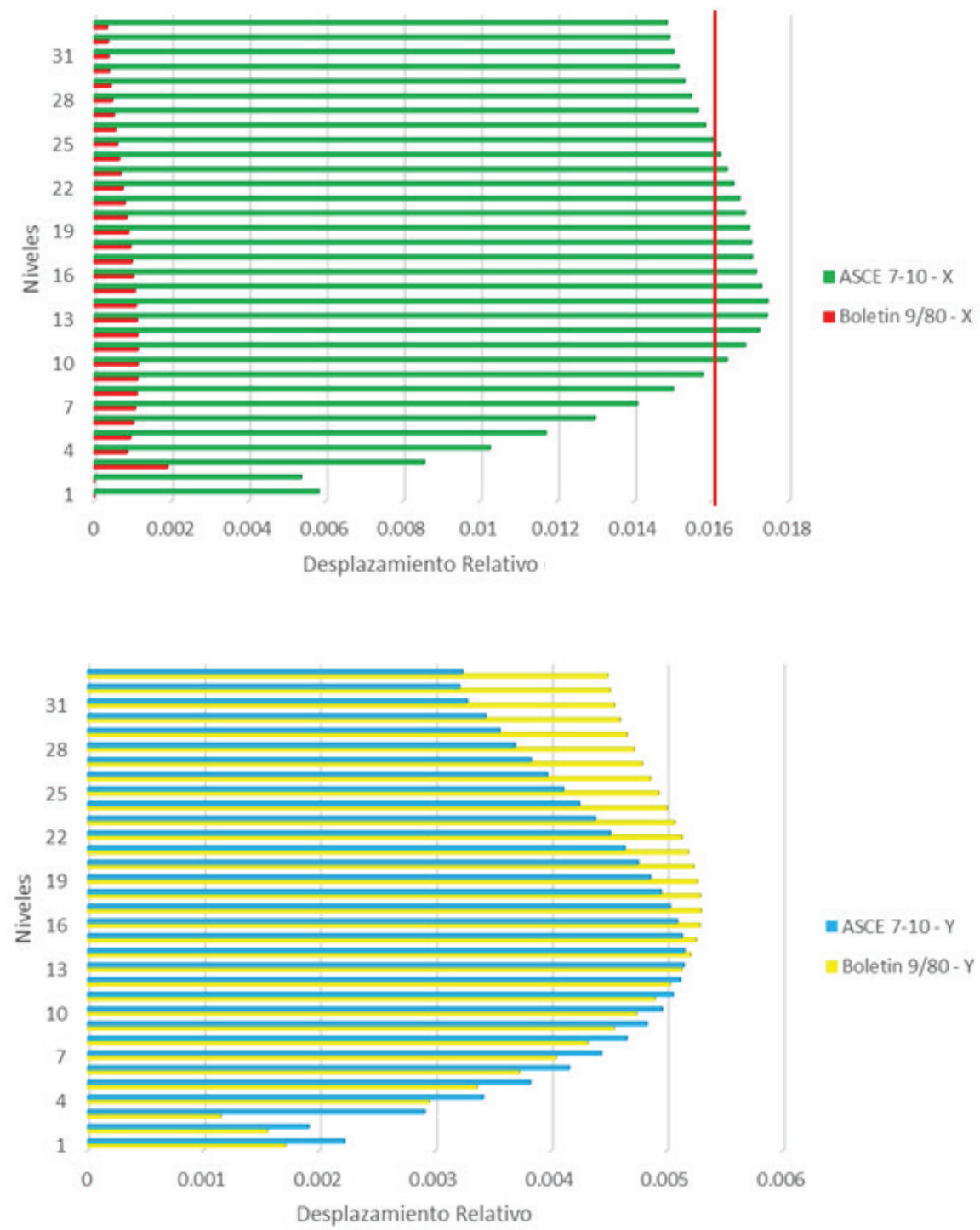

Figura 14. Derivas de entrepiso inducidas por viento estimadas de acuerdo al procedimiento direccional del ASCE 7-10 y la normativa vigente Boletín 9/80 


\section{Conclusiones}

Este artículo presentó los resultados de un análisis de las demandas estructurales inducidas por vientos huracanados y terremotos en un edificio flexible diseñado para la ciudad de Santo Domingo en la República Dominicana. La estructura considerada en este artículo es un edificio de 33 niveles con periodos fundamentales de vibración mayores de 3 segundos en las dos principales direcciones ortogonales, por lo que se considera un edificio flexible. El edificio fue inicialmente diseñado para cumplir con los requerimientos de la normativa vigente para efectos inducidos por terremotos (R-001) sin tomar en consideración los efectos inducidos por vientos huracanados; subsecuentemente, las demandas inducidas por viento fueron estimadas usando el procedimiento direccional del ASCE 07-10, utilizando como base la velocidad de diseño determinada en recientes mapas de amenaza de viento desarrollados para la región del Caribe.

De acuerdo a los resultados de los análisis realizados se concluye que los efectos inducidos por vientos huracanados son significativamente mayores a los efectos sísmicos por factores que oscilan entre 3 y 10, lo que muestra la urgencia de actualizar la actual normativa que define las cargas mínimas de diseño por viento de la República Dominicana. Las demandas estructurales comparadas incluyeron desplazamientos laterales, derivas de entrepiso, cortante basal y momento de vuelco. Además de mostrar que las demandas de viento exceden significativamente las de sismo, las demandas estimadas de acuerdo al procedimiento direccional del ASCE 07-10 resultaron en demandas mayores que las estimadas con la normativa vigente (Boletín 9/80) excediendo las mismas por factores entre 2 y 3 .

\section{Reconocimientos}

Esta investigación fue parcialmente apoyada por el proyecto de investigación del Instituto Tecnológico de Santo Domingo (INTEC) y del Ministerio de Educación Superior Ciencia y Tecnología (MESCYT) a través del programa FONDOCYT. Los autores agradecen el soporte de ambas instituciones en esta investigación. Los autores agradecen 
de manera especial al Ing. Luis Abbott Zorrilla por facilitar el modelo computacional de la estructura analizada.

\section{Referencias}

American Society of Civil Engineers (2010). Minimum Design Loads for Buildings and Other Structures (ASCE 7-10).

Billah, K., Scanlan, R. (1990). Resonance, Tacoma Narrows bridge failure, and undergraduate physics textbooks. American Journal of Physics, 59(2), 118.

Calais, E., Mazabraud, B., Mercier de Le' pinay, Mann, P., Mattioli, G., \& Jansma, P. (2002). Strain partitioning and fault slip rates in the northeastern Caribbean from GPS measurements. Geophysical Research Letters, 29(18), 1856. Doi: 10.1029/2002GL015397

Erazo, K. (2019). Probabilistic seismic hazard analysis and design earthquake for Santiago, Dominican Republic. Ciencia, Ingenierias Y Aplicaciones, 2(1), 67-84. Disponible en https://doi. org/10.22206/cyap.2019.v2i1.pp67-84

Erazo, K. (2020). Análisis probabilístico de peligro sísmico y terremoto de diseño para Santiago-República Dominicana. Ciencia, Ingenierias Y Aplicaciones, 3(1), 7-30. Disponible en https://doi. org/10.22206/cyap.2020.v3i1.pp7-30

López, R. \& Martínez, J. (2003). Daños observados en Puerto Plata, República Dominicana, causados por el terremoto del 22 de septiembre de 2003. Revista Internacional de Desastres Naturales, Accidentes e Infraestructura Civil, 3(2), 189-204.

Ministerio de Economía, Planificación y Desarrollo (2012). Amenazas y Riesgos Naturales en la República Dominicana, Compendio de Mapas. Santo Domingo, República Dominicana.

Ministerio de Obras Públicas y Comunicaciones (1980). Recomendaciones provisionales para el análisis por viento de estructuras Boletín No. $9 / 80$.

Ministerio de Obras Públicas y Comunicaciones (2011). Reglamento para el análisis y diseño sismico de estructuras, R-001. Dirección General de Reglamentos y Sistemas. Santo Domingo, D.N., República Dominicana. 
Neris, K., Domínguez, J., Pérez, J., Rodríguez, B. \& Cano, E. (2010). Control de la edificación como medida de reducción de riesgo al desastre causado por sismos en la República Dominicana. Chile: Congreso Iberoamericano de Ingeniería de proyectos.

Rozas, P. \& Sánchez, R. (2004). Desarrollo de infraestructura y crecimiento económico: revisión conceptual. CEPAL, División de Recursos Naturales e Infraestructura, serie 75.

Vickery, P.J. \& Wadhera, D. (2010). Development of Design Wind Speed Maps for the Caribbean for Application with the Wind Load Provisions of ASCE 7. Pan American Health Organization, World Health Organization. 